\title{
CONSENTIMIENTO INFORMADO EN LA RECIENTE REGULACIÓN DE LA TELEMEDICINA EN URUGUAY
}

\author{
INFORMED CONSENT IN THE RECENT REGULATION \\ OF TELEMEDICINE IN URUGUAY
}

\section{CONSENTIMENTO INFORMADO NO RECENTE REGULAMENTO DA TELEMEDICINA NO URUGUAI}

\author{
Diego Andrade Sosa * \\ Valeria Escobar Suhr **
}

RESUMEN. En el presente artículo se analizarán algunos de los aspectos medulares de la Ley $N^{\circ} 19.869$ (a un año de su entrada en vigencia), que a texto expreso reguló en nuestro país los servicios provistos mediante Telemedicina; y principalmente en lo atinente a la formación del consentimiento informado a través de dicha práctica.

PALABRAS CLAVE. Telemedicina. Datos Personales. Autonomía. Consentimiento Informado. Reglamentación.

ABSTRACT. This paper will analyze some of the core aspects of Law No. 19,869 (one year after its entry into force), which expressly regulated in our country the services provided through Telemedicine; and mainly with regard to the formation of informed consent through such practice.

KEY WORDS. Telemedicine. Personal Data. Autonomy. Informed Consent. Regulation.

RESUMO. Este artigo analisará alguns dos aspectos centrais da Lei No 19.869 (um ano após da sua entrada em vigor), que por meio de texto expresso regulamentou em nosso país os serviços prestados por meio da Telemedicina; e principalmente no que diz respeito à formação do consentimento informado por meio dessa prática.

\footnotetext{
* Doctor en Derecho y Ciencias Sociales. Asesor Legal del Hospital Pasteur, del Hospital de Canelones "Dr. Francisco Soca" y del Hospital de Las Piedras "Dr. Alfonso Espínola” de A.S.S.E. Ayudante (G1) de Derecho Civil en la Facultad de Ciencias Económicas y de Administración de la UDELAR. Aspirante a Profesor Adscripto de Derecho del Trabajo y de la Seguridad Social en la Facultad de Derecho UDELAR. Integrante del Grupo de Estudio Bioética y Derecho de la Facultad de Derecho de la UDELAR. Maestrando en Derecho del Trabajo y de la Seguridad Social Escuela de Posgrado UDELAR. Correo electrónico: dandradesosa@gmail.com ** Doctora en Derecho y Ciencias Sociales. Asesora Legal del Hospital Español “Dr. Juan José Crottogini” y de SAMME. A.S.S.E. Docente de Derecho Médico en el IEEM. Asistente (G2) de Derecho Civil en la Facultad de Ciencias Económicas y de Administración de la UDELAR. (G1) de Introducción a la Bioética y de Derecho Privado IV y V en la Facultad de Derecho de la Universidad de la República. Integrante del Grupo de Estudio Bioética y Derecho de la Facultad de Derecho de la UDELAR. Maestranda en Derecho Comercial Escuela de Posgrado UDELAR. Correo electrónico: vescobarsuhr@gmail.com
} 
PALAVRAS CHAVE. Telemedicina. Dados Pessoais. Autonomia. Consentimento Informado. Regulamento.

\section{Introducción.}

El contexto actual de pandemia global originado por el COVID-19, ha motivado el surgimiento y consolidación de determinados medios telemáticos que permiten continuar desempeñando ciertas actividades, evitando la presencialidad física, con la finalidad de detener la virulencia en los contagios del notorio patógeno.

En tal sentido, plataformas informáticas "a distancia" han viabilizado la continuidad, entre otros, de servicios económicos, educativos y profesionales del más amplio rango. Dentro de estos últimos encontramos a las prestaciones de salud, ya que, más allá de los servicios que constituyen el primer frente de batalla contra la enfermedad, como lo son la Atención Domiciliaria, Emergencias, y Unidades de Medicina Intensiva; existen otros en los que se ha optado por una modalidad de cobertura asistencial médicopaciente no presencial, a fin de que los últimos no concurran innecesariamente a los centros sanitarios.

Precisamente, desde que se ha decretado la Emergencia Sanitaria en nuestro país, por el Decreto $N^{o} 92 / 020$ de 13/03/2020 se ha potenciado la utilización del recurso de la "telemedicina", el cual entendemos que claramente ha venido para quedarse.

Sin lugar a dudas, la utilización del mismo ha presentado una serie de ventajas en materia de prestación de asistencia; en especial porque permite acortar distancias al permitir, por ejemplo, que aquellos centros de salud del interior del país que puedan no contar con determinadas especialidades médicas, acceder a una consulta; lo cual redunda en un beneficio innegable para el paciente, y en definitiva proporciona al Sistema de Salud un insumo de palmaria eficiencia y efectividad. Pensemos, entre otras potenciales ventajas en la posibilidad para usuarios de poblaciones ubicadas en parajes recónditos de Uruguay y con dificultades en el acceso en el transporte a los centros urbanos de mayor importancia; que ahora tienen la posibilidad a los más destacados especialistas médicos nacionales, quienes generalmente se encuentran afincados en el segundo tipo de lugar referido.

Asimismo, resulta menester señalar que esta vía de prestación cuenta con una novel regulación en nuestro ordenamiento jurídico, a través de la Ley $\mathrm{N}^{\circ} 19.869$ de 02/04/2020; lo cual y a pesar de significar un avance incuestionable, conlleva a que surjan en la práctica una serie de dificultades en relación a la forma de implementación de la misma, a fin de impedir la vulneración de derechos fundamentales de los pacientes 
En dicha línea cabe recordar que el desenvolvimiento y vicisitudes de la actuación asistencial, en muchas ocasiones, como es sabido, se requerirá recabar por parte del profesional médico el consentimiento informado del paciente. La prestación de la atención sanitaria mediante el recurso de la "telemedicina" impide la inmediatez con el paciente a fin de recabar dicho consentimiento; lo que en definitiva importa una complejidad, respecto de la modalidad de resguardar dicho pronunciamiento.

Sobre lo mencionado en el párrafo anterior, sumado a un somero análisis del articulado de la aludida Ley y algunas propuestas que modestamente entendemos que podrían ser consideradas para el caso de que en lo sucesivo se proceda, por parte del Poder Ejecutivo, a efectuar una reglamentación de la Ley de marras.

Como necesaria aclaración preliminar se expresa que los comentarios y opiniones vertidas en este artículo son de estricta responsabilidad de los autores y no comprometen a las instituciones donde los mismos se desempeñan.

Asimismo, recomendamos al lector que tenga interés en profundizar en los contenidos de los diferentes cuerpos normativos de origen uruguayo a los que se hará alusión en el presente trabajo, que acuda al sitio web del Diario Oficial (https://www. impo.com.uy) a fin de acceder a las versiones íntegras, vigentes y actualizadas de los mismos.

\section{Generalidades de la Ley $N^{\circ}$ 19.869: la incipiente regulación de la Telemedicina en Uruguay.}

La Ley $\mathrm{N}^{\circ} 19.869$ de 02/04/2020, constituye la primera versión de la normativa uruguaya tendiente a regular la novel modalidad de prestación de servicios médicos; y como lo indica su artículo 1 tiene por objeto establecer los lineamientos generales para la implementación y desarrollo de la telemedicina como prestación de los servicios de salud, a fin de mejorar su eficiencia, calidad e incrementar su cobertura mediante el uso de tecnologías de la información y de la comunicación.

En el presente capítulo aludiremos a los aspectos que estimamos como más relevantes del articulado; exceptuando lo atinente al consentimiento informado, a cuyo desarrollo abocaremos el capítulo siguiente.

A los efectos conceptuales, el artículo 2 del cuerpo normativo de marras, define a la telemedicina como "la provisión de los servicios de atención sanitaria, donde la distancia es un factor crítico, por todos los profesionales de atención sanitaria utilizando tecnologías de la información y comunicación para el intercambio de información válida 
para el diagnóstico, tratamiento y prevención de enfermedades y lesiones, investigación y evaluación, y para la educación continua de los proveedores de atención sanitaria, todo en interés de mejorar la salud de sus individuos y sus comunidades". Sin perjuicio de lo expresado, los artículos 4 y 5, establecen que se considerarán como servicios de telemedicina "todos aquellos reconocidos como tales por el Ministerio de Salud Pública", y declaran, con carácter interpretativo que la Ley $\mathrm{N}^{\circ} 18.335$ de 15/08/2008 sobre "Derechos y Obligaciones de Pacientes y Usuarios de los Servicios de Salud" comprende las prestaciones médicas llevadas a cabo mediante el uso de telemedicina.

La actividad regulada se sustenta en base a una serie de principios que son enunciados y definidos por el artículo 3 de la Ley, a saber:

“A) Universalidad. - A través de la telemedicina se garantiza un mejor acceso de toda la población a los servicios de salud.

B) Equidad. - La telemedicina permite derribar barreras geográficas, acercando los servicios a la población en lugares remotos y con escasez de recursos.

C) Calidad del servicio. - La telemedicina promueve una mejora en la calidad y atención integral del paciente, fortaleciendo las capacidades del personal de salud.

D) Eficiencia. - La telemedicina permite optimizar los recursos asistenciales, la mejora en la gestión de la demanda, la reducción de las estancias hospitalarias, la disminución de la repetición de actos médicos y los desplazamientos a través de la comunicación de los profesionales.

E) Descentralización. - La telemedicina es una estrategia de utilización de recursos sanitarios que optimiza la atención en los servicios de salud fortaleciendo el proceso de descentralización del Sistema Nacional Integrado de Salud.

F) Complementariedad. - El ejercicio clínico de la medicina requiere el vínculo directo con el paciente. La telemedicina es un complemento a la asistencia brindada por el médico tratante (artículo 24 de la Ley $N^{\circ}$ 19.286, de 25 de setiembre de 2014).

G) Confidencialidad.- Se debe preservar la confidencialidad en la relación médicopaciente, garantizando la seguridad en el intercambio de información entre profesionales o centros de atención sanitaria". 
En el artículo 4 se establece que se consideran servicios de telemedicina todos aquellos reconocidos como tales por el Ministerio de Salud Pública; al cual se faculta para dictar los protocolos de actuación para cada uno de los servicios de telemedicina en el plazo de noventa desde la promulgación de la ley.

Por su parte el artículo 5 declara con tenor interpretativo que la Ley $\mathrm{N}^{\circ} 18.335$ de 15/08/2008 (sobre Derechos y Deberes de los Pacientes y Usuarios de los Servicios de Salud), comprende las prestaciones médicas llevadas a cabo mediante el uso de telemedicina.

En el artículo 6 prevé que los servicios de salud podrán ofrecer a sus usuarios, servicios de telemedicina, brindando información pormenorizada al respecto; a cuyos efectos deberán contar con el personal adecuado y la infraestructura necesaria, quedando sometidos a lo previsto por los artículos 157 a 160 de la Ley $N^{\circ} 18.719$ de 27/12/2010. Dichos artículos citados refieren a las potestades de contralor y colaboración de la Agencia para el Desarrollo del Gobierno de Gestión Electrónica y la Sociedad de la Información y del Conocimiento en materia de intercambio de información entre entidades públicas, sean estatales o no.

Precisamente en materia de transmisión y almacenamiento de datos e información personal mediante el uso de telemedicina, el artículo 8 de la Ley $\mathrm{N}^{\circ} 19.869$, en la redacción dada por el artículo 397 de la Ley No 19.924 de 18/12/2020 establece que los mismos serán tratados de conformidad con lo establecido en la Ley $N^{\circ} 18.331$ (Ley de Protección de Datos Personales), de 11/08/2008; delegándose a la reglamentación la determinación de las medidas de seguridad y responsabilidad proactiva según el tipo de dato, tratamiento y sujetos involucrados.

Evidentemente la remisión efectuada a la Ley de Protección de Datos Personales, no resulta baladí en la materia; y amerita efectuar un detenimiento sobre los alcances de dicho cuerpo normativo en materia de información sanitaria.

Así la Ley $\mathrm{N}^{\circ} 18.331$ de 11/08/2008; comienza estableciendo, en su artículo $1^{\circ}$, que "El derecho a la protección de datos personales es inherente a la persona humana, por lo que está comprendido en el artículo 72 de la Constitución de la República"; consagrándose en el artículo 11 un principio de reserva, en virtud del cual las personas físicas o jurídicas que obtuvieren legítimamente información proveniente de una base de datos que les brinde tratamiento, están obligadas a utilizarla en forma reservada y exclusivamente para las operaciones habituales de su giro o actividad, estando prohibida toda difusión de la misma a terceros; así como a guardar estricto secreto profesional sobre 
los mismos (artículo 302 del Código Penal), cuando hayan sido recogidos de fuentes no accesibles al público; excepto en los casos de orden de la Justicia competente o si mediare consentimiento del titular.

Por otra parte, en literal "E" del artículo 4 se califican como "datos sensibles" las informaciones relativas a la salud de las personas. Los efectos que dicha categorización importan, se encuentran explicitados en el artículo 18 de la Ley; destacándose, dentro de otros aspectos, y a los siguientes: 1) que ninguna persona puede ser obligada a proporcionar datos sensibles; 2) que dichos datos sólo podrán ser objeto de tratamiento con el consentimiento expreso y escrito del titular;3) que los datos sensibles sólo pueden ser recolectados y objeto de tratamiento cuando medien razones de interés general autorizadas por ley, o cuando el organismo solicitante tenga mandato legal para hacerlo. También podrán ser tratados con finalidades estadísticas o científicas cuando se disocien de sus titulares.

Concomitantemente el artículo 19 establece que "Los establecimientos sanitarios públicos o privados y los profesionales vinculados a las ciencias de la salud pueden recolectar y tratar los datos personales relativos a la salud física o mental de los pacientes que acudan a los mismos o que estén o hubieren estado bajo tratamiento de aquéllos, respetando los principios del secreto profesional, la normativa específica y lo establecido en la presente ley". Dicha previsión debe ser complementada con el Principio de reserva consagrado en el artículo 11, el cual establece que: "Aquellas personas físicas o jurídicas que obtuvieren legítimamente información proveniente de una base de datos que les brinde tratamiento, están obligadas a utilizarla en forma reservada y exclusivamente para las operaciones habituales de su giro o actividad, estando prohibida toda difusión de la misma a terceros. Las personas que, por su situación laboral u otra forma de relación con el responsable de una base de datos, tuvieren acceso o intervengan en cualquier fase del tratamiento de datos personales, están obligadas a guardar estricto secreto profesional sobre los mismos (artículo 302 del Código Penal), cuando hayan sido recogidos de fuentes no accesibles al público. Lo previsto no será de aplicación en los casos de orden de la Justicia competente, de acuerdo con las normas vigentes en esta materia o si mediare consentimiento del titular. Esta obligación subsistirá aun después de finalizada la relación con el responsable de la base de datos".

Debe por último destacarse que el artículo 35 de la Ley, en la redacción dada por los artículos 152 de la Ley No 18.719 de 27/12/2010 y 83 de la Ley No 19.355 de 19/12/2015, confiere a la Unidad Reguladora y de Control de Datos Personales de la Agencia para el Desarrollo del Gobierno de Gestión Electrónica y la Sociedad de la Información y del 
Conocimiento (AGESIC) la posibilidad de aplicar sanciones a los responsables de las bases de datos, encargados de tratamiento de datos personales y demás sujetos alcanzados por el régimen legal que infrinjan las obligaciones de reserva anteriormente referenciadas.

Cabe traer a colación que, en el ámbito sanitario, el conocimiento de datos, hechos, actuaciones e incluso secretos de las personas, conjuntamente con la exhibición de determinadas partes de su cuerpo, resulta un requisito ineludible para que se propicie la atención médica. Esta situación hace surgir, según BERRO y ADRIASOLA una confidencialidad "necesaria sobre la vida íntima de la persona enferma, por lo que debe garantizarse la reserva, el secreto médico. La intimidad a pesar de la intromisión de médicos y de otros profesionales del equipo asistencial debe quedar incólume" (BERRO y ADRIASOLA, 2013, 396). FRANÇA, citada por GIL YACOBAZZO y VIEGA RODRÍGUEZ, define a dicha regla de confidencialidad como "el derecho de toda persona a proteger los datos que le pertenecen en virtud de que los considera reservados o restringidos (por pertenecer a la intimidad) o su derecho a limitar la difusión de ciertas informaciones $u$ opiniones emitidas" (GIL YACOBAZZO y VIEGA RODRÍGUEZ, 2018, 229).

Lo anteriormente expuesto nos lleva en forma inevitable a sostener que la información relativa al estado de salud debe ser categorizada como una especie dentro del género "derecho a la intimidad" y en definitiva como un derecho fundamental.

La disgresión de los párrafos antecedentes apunta a subrayar la responsabilidad del prestador en caso de que a través de los medios tecnológicos que viabilicen la telemedicina se producen violaciones indebidas a la intimidad sanitaria del paciente. Debemos recordar que tales infracciones que generen perjuicios en los usuarios, son generadores de responsabilidad civil contractual para la institución asistencial. Sin perjuicio de que la alusión efectuada excede los objetivos del presente artículo, nos remitimos a postura formulada en reciente trabajo de los autores, en el que se concluye que las instituciones de asistencia médica son verdaderos proveedores de servicios, y como contrapartida sus afiliados los consumidores de dicho servicio; entablándose entre ambos una verdadera relación consumo, susceptible de ser regulada por la Ley $\mathrm{N}^{\circ} 17.250$ "De relaciones de consumo y defensa del consumidor" de 11/08/2000 (en lo sucesivo LRC); y por deducción hermenéutica derivada de una relación género-especie, que determina que mediante la telemedicina se desarrollen prestaciones médico-asistenciales, debemos sostener, sin hesitaciones, que la misma también resulta susceptible de regulación por la LRC. (ESCOBAR SUHR y ANDRADE SOSA, 2020, 158) 
Finalmente, retomando el análisis general de la Ley $\mathrm{N}^{\circ} 19.869$, en el artículo 9 se establece que las consultas o intercambios de información que se realicen mediante el uso de telemedicina con profesionales o instituciones de salud residentes en el extranjero, estarán alcanzados por las disposiciones de la ley y por las demás normas reglamentarias para la prestación del servicio.

Debe destacarse que al día de la elaboración del presente trabajo, la Reglamentación de la Ley No 19.869 por parte del Poder Ejecutivo se encuentra pendiente. Entendemos que el acaecimiento de la misma resultará fundamental para aterrizar en la vía práctica algunas cuestiones elementales de la ejecución de la prestación del servicio. A modo ilustrativo será de especial relevancia, en nuestro concepto, la regulación de la transmisión de información, mediante mecanismos que garanticen una impenetrable confidencialidad.

\section{La formación del consentimiento informado en la telemedicina}

\section{autonomía.}

3.1. El consentimiento informado como materialización del principio de

El principio de autonomía regente en la toma de decisiones sanitarias, es indubitablemente la piedra angular sobre la que se ha edificado el vigente modelo de relación médico-paciente, denominado precisamente como autonómico, superador del paternalismo (modelo este último en el que el paciente no ejerce ningún derecho de autodeterminación sino que queda subordinado a lo que dispone el médico, el cual se entiende que en todo caso actuará en beneficio del paciente, decidiendo por él en lo que desconoce.

ORDOQUI CASTILLA define a la autonomía del paciente como "la capacidad de decidir libremente con relación a las sugerencias que le realiza el médico con referencia a los tratamientos a seguir en el proceso de curación de su enfermedad. Sin autonomía, sin libertad no puede haber consentimiento informado" (ORDOQUI CASTILLA, 2012, 7); agregando luego que "Una persona autónoma es la que actúa con libertad según sus propias convicciones e intereses” (ORDOQUI CASTILLA, 2012, 7).

En otras palabras, el respeto de la autonomía en el ámbito médico es una obligación de los profesionales de la salud para asegurar que los pacientes tengan derecho a escoger. Para que el profesional de la salud pueda afirmar que las decisiones de los pacientes son autónomas debe estar seguro que son informadas, libres, voluntarias y auténticas. 
Anivel de los instrumentos internacionales, en el artículo 5 de la Declaración Universal sobre Bioética y Derechos Humanos de UNESCO (en lo sucesivo DUBDH) de 19/10/2005, encontramos un recogimiento de este principio con perspectivas globales al señalar que "Se habrá de respetar la autonomía de la persona en lo que se refiere a la facultad de adoptar decisiones, asumiendo la responsabilidad de éstas y respetando la autonomía de los demás. Para las personas que carecen de la capacidad de ejercer su autonomía, se habrán de tomar medidas especiales para proteger sus derechos e intereses".

Este derecho a la autonomía es reconocido en forma genérica e irrestricta a todas las personas, sin distinción. Particularmente, una de sus materializaciones o corolarios más importantes, es el consentimiento informado es reconocido tanto en materia clínica como de investigación en seres humanos, en sendos compendios tales como la Ley $\mathrm{N}^{\mathrm{o}} 18.335$ de 15/08/2008 y su Decreto Reglamentario No 274/010 de 08/09/2010 (Derechos y Deberes de los Usuarios), Ley No 18.473 de 03/04/2009 (Voluntades Anticipadas), Ley $N^{\circ} 19.286$ de 25/09/2014 (Código de Ética Médica), Decreto No 158/019 de 03/06/2019 (Investigación en Seres Humanos).

En tal sentido, ORDOQUI CASTILLA refiere que:

"el consentimiento informado (asentimiento) es el ejercicio del derecho en forma unilateral por una persona capaz que forma parte directa o indirecta de la relación médico-paciente que acepta sugerencia de intervención médica a partir de una decisión libre después de haber recibido información clara, completa, continua, oportuna previa. El consentimiento informado es un verdadero proceso en que se pasan distintas etapas: el médico informa; el paciente se informa, el médico sugiere, el paciente acepta" (ORDOQUI CASTILLA, 2012, 43)

El paciente ejerce su autonomía y el profesional deberá obtenerlo en todas aquellas circunstancias que no estén exceptuadas (razones de urgencia o emergencia, o de notoria fuerza mayor, y las circunstancias no permitan demora por existir riesgo grave para la salud del paciente, o cuando se esté frente a patologías que impliquen riesgo cierto para la sociedad que integra, tal como también se establece en el artículo 22 del decreto 274/2010, ya referido).

La obligatoriedad del otorgamiento del consentimiento informado en la relación médico-paciente, se encuentra regulado en el artículo 16 del decreto 274/2010 reglamentario de la ley $\mathrm{N}^{\mathrm{o}} 18.335$ referente a los Derechos y obligaciones de pacientes y usuarios de los Servicios de Salud reza: "El otorgamiento del consentimiento informado es un derecho del paciente y su obtención un deber del profesional de la salud" Y por su 
parte el artículo 11 de la citada Ley No 18335 señala que "Todo procedimiento de atención médica será acordado entre el paciente o su representante, luego de recibir información adecuada, suficiente y continua, y el profesional de salud. El consentimiento informado del paciente a someterse a procedimientos diagnósticos o terapéuticos estará consignado en la historia clínica en forma expresa. Este puede ser revocado en cualquier momento".

Como resulta apreciable la obtención del consentimiento informado constituye un presupuesto sine quanon para el desarrollo de una relación médico-paciente, respetuosa de los derechos fundamentales de las personas y con las debidas garantías para ambas partes. Sobre el particular, estimamos que la omisión del galeno en recabar dicha manifestación de voluntad de su paciente es fuente originadora de responsabilidad civil profesional, por incumplimiento de un deber integrante del íter constitutivo de las obligaciones de medios que asumen los médicos para el desempeño de su profesión.

\subsection{El consentimiento informado en la Ley $N^{o} 19.869$.}

En su artículo 7 la mentada Ley refiere al consentimiento informado, realizando primeramente la siguiente aseveración: "Para brindar servicios de telemedicina, los servicios de salud deberán recabar el consentimiento expreso del paciente por cada acto médico a realizarse, quedando sujetos a lo previsto en el artículo 11 de la Ley $N^{\circ} 18.335$, de 15 de agosto de 2008".

La previsión enunciada deriva de una obviedad cuasi silogística; en tanto a través de los servicios de telemedicina se efectúan prestaciones asistenciales para cuya ejecución legamente válida es necesario el consentimiento invocado; a fin de acreditar la existencia de una decisión verdaderamente autónoma por parte del usuario o paciente.

Posteriormente el artículo agrega "El paciente deberá otorgar consentimiento expreso para la realización de tratamientos, procedimientos, diagnósticos, así como la transmisión e intercambio de la información personal que se desprenda de su historia clínica, con las limitaciones previstas en el literal D) del artículo 18 de la Ley $N^{\circ} 18.335^{\prime \prime}$. En este punto, el articulado también sigue los lineamientos generales de la normativa patria en la materia, tanto de consentimiento, como del manejo de los datos sensibles sanitarios.

Con respecto a la primera parte, es dable hacer referencia a los supuestos que habilitan, en el orbe positivo uruguayo, a la prescindencia del consentimiento informado. En tal sentido es el tercer inciso del artículo 11 de la Ley $\mathrm{N}^{\circ} 18.335$, que habla de dicha situación excepcional: "Cuando mediaren razones de urgencia o emergencia, o de notoria fuerza mayor que imposibiliten el acuerdo requerido, o cuando las circunstancias no 
permitan demora por existir riesgo grave para la salud del paciente, o cuando se esté frente a patologías que impliquen riesgo cierto para la sociedad que integra, se podrán llevar adelante los procedimientos, de todo lo cual se dejará precisa constancia en la historia clínica".

Pareciera, prima facie, que el ámbito de la telemedicina resultaría más propenso o habitual en su uso al tipo de consulta coordinada o de policlínica, por que no aportaría una plataforma fáctica habilitante del aludido apartamiento. Pero, la normativa aprobada no impide la utilización de tales servicios en los supuestos de urgencia o emergencia. De hecho entendemos que el empleo del mecanismo en tales situaciones puede ser de especial utilidad, no solamente cuando acuda directamente al mismo el usuario o paciente, sino por ejemplo, en la realización de una interconsulta remota a un especialista desde un Servicio de Emergencia "presencial". Consecuentemente la invocación de la excepción sería plausible, en tanto se den los supuestos específicos establecidos por la norma; y siempre bajo la égida de que la obtención del consentimiento informado del paciente es la regla y su ausencia una notoria excepción, derivada de circunstancias imponderables.

Continuando con el análisis del artículo 7, en su tercer inciso se señala: "El consentimiento (...) puede ser revocado por el paciente en cualquier momento. La revocación surtirá efectos desde su comunicación fehaciente al servicio de salud". En este aspecto nuevamente se destaca la coherencia sistémica sobre la regulación de este particular, ya que la posibilidad de revocar el consentimiento oportunamente brindado, ha sido recogido en sendos textos legales uruguayos, tanto en sede de atención médica como de investigación en seres humanos (Leyes $\mathrm{N}^{\circ} 18.335, \mathrm{~N}^{\circ} 18.473,19.286$, Decreto $\mathrm{N}^{\circ}$ 274/010, Decreto $\left.\mathrm{N}^{\circ} 158 / 019\right)$, en tanto es un corolario evidente del principio de autonomía, reconocido también a nivel universal. Así la DUBDH en su artículo 6 establece: "Toda intervención médica preventiva, diagnóstica y terapéutica sólo habrá de llevarse a cabo previo consentimiento libre e informado de la persona interesada, basado en la información adecuada. Cuando proceda, el consentimiento debería ser expreso y la persona interesada podrá revocarlo en todo momento y por cualquier motivo, sin que esto entrañe para ella desventaja o perjuicio alguno".

Finalmente el último inciso del artículo 7 señala "En el caso de que el paciente sea menor de edad o persona declarada legalmente incapaz, el artículo será aplicable a su tutor o representante legal".

En este aspecto la norma citada pareciera adoptar un criterio clásico civilista, en el que la voluntad del menor de edad o del incapaz declarado únicamente puede ser manifestada por el representante legal del mismo; soslayando (reiteramos en apariencia) 
algunos desarrollos bioético jurídicos sobre el particular. A vía ilustrativa, en relación a los menores de edad maduros, la normativa uruguaya reconoce el principio de autonomía progresiva respecto a sus decisiones en el ámbito sanitario. Así, el artículo 11-BIS del Código de la Niñez y de la Adolescencia (aprobado por la Ley No 17.823 de 07/09/2004), establece, en materia de información y acceso a los servicios de salud, que "De acuerdo a la edad del niño, niña o adolescente se propenderá a que las decisiones sobre métodos de prevención de la salud sexual u otros tratamientos médicos que pudieran corresponder, se adopten en concurrencia con sus padres $u$ otros referentes adultos de su confianza, debiendo respetarse en todo caso la autonomía progresiva de los adolescentes"; añadiendo posteriormente que "En caso de existir riesgo grave para la salud del niño, niña o adolescente y no pudiera llegarse a un acuerdo con éste o con sus padres o responsables del mismo en cuanto al tratamiento a seguir, el profesional podrá solicitar el aval del Juez competente en materia de derechos vulnerados o amenazados de niños, niñas y adolescentes, quien a tales efectos deberá recabar la opinión del niño, niña o adolescente, siempre que sea posible".

En la misma línea que la norma aludida en el párrafo que antecede, encontramos al artículo 11 del Decreto $N^{0} 274 / 010$ de 08/09/2010, Reglamentario de la precitada Ley $\mathrm{N}^{0} 18.335$, que señala que: "Los adolescentes a quienes, de acuerdo al principio de autonomía progresiva, los profesionales de la salud consideren suficientemente maduros para recibir atención fuera de la presencia de los padres, tutores u otros responsables, tienen derecho a la intimidad y pueden solicitar servicios confidenciales e incluso tratamiento confidencial".

Con relación a la situación de los incapaces mayores de edad, entendida estrictamente en un sentido jurídico, como la imposibilidad total de tener un conocimiento cabal de su situación y de manifestar libremente su voluntad (hipótesis en las que los textos legales prevén como necesaria la designación de un curador); debe sostenerse, según GÓMEZ SÁNCHEZ “que el sujeto no reúne las condiciones necesarias para ser titular del derecho a no saber" (GÓMEZ SÁNCHEZ, 2016).

No obstante, la constitucionalista ibérica de marras también alude a la situación de otros tipos de "incapacidades" de grado inferior. Así, en el caso de incapacidades físicas, tales como la sordera o la ceguera que pueden "ser suplidas con medios materiales el sujeto es titular del derecho a no saber y, por tanto, el criterio debería ser el de utilizar los medios técnicos que permitan superar dicha discapacidad y hacer posible la manifestación de voluntad" (GÓMEZ SÁNCHEZ, 2016). Por otra parte, si se tratara de una disminución de la capacidad mental que no anule totalmente la voluntad del sujeto "el criterio debería ser similar al caso de los menores de edad: es decir, deberían ser 
oídos y su voluntad ser atendida cuando ello no fuera manifiestamente incompatible con su propio interés" (GÓMEZ SÁNCHEZ, 2016).

Los criterios esbozados en el párrafo anterior, se evidencian en determinadas fuentes normativas nacionales. En tal sentido, el artículo 23 de la Ley $\mathrm{N}^{\circ} 19.529$ de 24/08/2017 sobre "Salud Mental", establece que "Las personas con discapacidad tienen derecho a recibir la información a través de los medios y tecnologías adecuadas para su comprensión".

En definitiva, en este punto estimamos que debería esta efectuarse un cambio en la redacción del artículo, a fin de que el mismo mantenga una coherencia sistémica. Dicho cambio debería operar en el sentido que, sin desconocer el rol fundamental de los representantes de aquellas personas que no pueden manifestar plenamente una voluntad válida, por detentar alguna especie de incapacidad legal (derivada de su menor edad o condiciones mentales), se reconozca, pondere y empodere a estos últimos sujetos, para que, en la medida de sus facultades de discernimiento y comprensión de la realidad, puedan manifestar su consentimiento. Este último podrá propiciarse sea en la forma de asentimiento complementario de la voluntad de sus responsables; o sea en la forma de un verdadero consentimiento, si la madurez de la persona así lo permite, complementado por el conocimiento de la situación por parte de su representante legal.

\section{Aspectos a considerar en una eventual reglamentación de la Ley $\mathbf{N}^{\circ} 19.869$} en materia de consentimiento informado.

Si bien es indudable que el Derecho Positivo Sanitario uruguayo ha generado un avance insoslayable al integrar a su mapa un cuerpo normativo que consagra las bases fundamentales para la regulación de una nueva modalidad de prestación de servicios médicos en una vía telemática y a distancia. Asimismo estamos convencidos de que la aprobación de la Ley $\mathrm{N}^{\mathrm{o}} 19.869$ constituye el inicio de un camino, en el que auguramos la anexión de otras normas de igual o inferior rango, que complementen a aquella, añadiendo mayores especificaciones que indubitablemente serán necesarias ante una realidad claramente dinámica y que se encuentra atada a los cambios en el desarrollo tecnológicos.

No obstante, obran algunos aspectos indefinidos que estimamos deberían ser considerados en una eventual reglamentación de la Ley por parte del Poder Ejecutivo, que no solamente resultan atinentes a cuestiones prácticas, sino que serían tendientes a la protección de derechos fundamentales y al otorgamiento de garantías para ambas partes de la relación asistencial. 
Una de esas cuestiones es la forma en la que se recabará el consentimiento infirmado al que alude el artículo 7 del cuerpo normativo de marras; teniéndose presente que el mismo será efectuado enteramente a distancia, ya que tanto paciente como médico se encontrarán en entornos físicos diversos durante la realización de la consulta; y no se establece el medio o manera en que quedará documentado dicho pronunciamiento.

En nuestro concepto, las bases sobre las que se debe reglamentar este punto, deben apuntar a la existencia de un mecanismo que permita tanto a paciente como médico detentar un medio que acredite plenamente que existió el visto bueno del primero a la propuesta terapéutica asistencial del segundo; independientemente de que no figure en el tradicional soporte papel. En esta línea, siempre desde una perspectiva orgánica y coherente, y por razones de analogía, entendemos que debemos apuntar primeramente a la existencia de normas nacionales que admiten el registro y manejo de información sobre datos sanitarios en forma no física o tradicional.

Así un primer mojón, a nivel general, lo encontramos en la Ley $\mathrm{N}^{\mathrm{o}} 18.600$ de 21/09/2009, en el que se reconoció la validez y admisibilidad jurídica del documento y firma electrónicos y reguló los aspectos relativos a la identificación digital de personas. Dicho cuerpo legal establece en su artículo 2 algunas definiciones que asimilamos de particular interés para el objeto del presente trabajo, tales como: a) "Certificado electrónico":documento electrónico firmado electrónicamente que da fe del vínculo entre el firmante o el titular del certificado y los datos de creación de la firma electrónica; b) "Dispositivo de creación de firma": componente informático que sirve para aplicar los datos de creación de firma; c) "Dispositivo de verificación de firma": componente informático que sirve para aplicar los datos de verificación de firma; d) "Documento electrónico o documento digital": representación digital de actos o hechos, con independencia del soporte utilizado para su fijación, almacenamiento o archivo; e) "Firma electrónica": los datos en forma electrónica anexos a un documento electrónico o asociados de manera lógica con el mismo, utilizados por el firmante como medio de identificación; f) "Firma electrónica avanzada": la firma electrónica que cumple los siguientes requisitos: 1) Requerir información de exclusivo conocimiento del firmante, permitiendo su identificación unívoca; 2) ser creada por medios que el firmante pueda mantener bajo su exclusivo control; 3) ser susceptible de verificación por terceros; 4) estar vinculada a un documento electrónico de tal modo que cualquier alteración subsiguiente en el mismo sea detectable; y 5) haber sido creada utilizando un dispositivo de creación de firma técnicamente seguro y confiable y estar basada en un certificado reconocido válido al momento de la firma; y g) "Firmante o signatario": persona que utiliza bajo su exclusivo control un certificado electrónico o un certificado reconocido para efectuar operaciones de firma electrónica o firma electrónica avanzada. 
A nuestro juicio, cualquier reglamentación en materia de consentimiento informado "a distancia" debería considerar la posibilidad de que la obtención de dicha manifestación de voluntad pueda quedar consignada mediante a través de un signo modalidad similar al de la firma electrónica avanzada; dotando tanto al paciente como a la institución asistencial de alguna especie de dispositivo o de código informático que permite un registro recíproco de tal pronunciamiento. De todos modos, entendemos que este mecanismo puede detentar ciertas dificultades en su ejecución práctica para el corto y mediano plazo; ya que entrañaría la realización de un despliegue e inversión de importancia no menor, tanto en materia de difusión, capacitación y compra de los insumos que sean necesarios.

Otra regulación de trascendencia en la materia, complementaria de la anterior, específica en materia sanitaria y con vocación de inspiración reguladora para la cuestión ventilada en este capítulo, es la recogida en materia de historia clínica electrónica.

El Sistema de Historia Clínica Electrónica Nacional, instrumentado a través de la plataforma de Historia Clínica Electrónica Nacional, fue en forma primigenia instaurado por el artículo 466 de la Ley No 19.355 de 19/12/2015; facultando al Poder Ejecutivo a determinar los mecanismos de intercambio de información clínica con fines asistenciales, asegurando la confidencialidad de la información en concordancia con la Ley N ${ }^{0} 18.331$. En la reglamentación de esta normativa, dada por el Decreto $N^{\circ} 242 / 017$ de 31/08/2017, se define a la Historia Clínica Electrónica: como "el conjunto integral de datos clínicos, sociales y económicos, referidos a la salud de una persona, desde su nacimiento hasta su muerte, procesados a través de medios electrónicos, siendo el equivalente funcional de la historia clínica papel"; y al Documento Clínico Electrónico como "la representación digital de un evento clínico o asistencial suscrito por un trabajador de la salud" (artículo 2); estableciendo la obligatoriedad de todos los prestadores de salud, públicos y privados de llevar una historia clínica electrónica de cada persona, siendo responsables de su completitud y seguridad de acuerdo con las normas legales vigentes. Asimismo se establece en el artículo 4 una serie de principios sobre los que deberá ajustarse la historia clínica electrónica, oficiantes como criterio interpretativo y que definitiva no difieren de los regentes para su homóloga de soporte papel, a saber: a) finalidad; b) veracidad; c) completitud; d) reserva; e) información; y f) accesibilidad. Más adelante, en el artículo 11 se establece el valor documentación auténtica, válida y admisible como medio probatorio, siempre que se encuentre firmada electrónicamente

Más adelante en el tiempo, el artículo 194 de la Ley № 19.670 de 15/10/2018, estableció que las personas quedarán incorporadas al Sistema de Historia Clínica Electrónica Nacional través de la registración de su información por parte de la entidad prestadora de servicios de salud; consagrando el derecho de toda persona, en cualquier momento, podrá 
oponerse al acceso a su información clínica a través de la Plataforma de Historia Clínica Electrónica Nacional, manifestación que será revocable. Dicha oposición impedirá el acceso a la información clínica, salvo en las excepciones legalmente previstas. La reglamentación de este artículo se verificó con el dictado del Decreto No 122/019 de 29/04/2019; en la que se establece, entre otros aspectos, como derechos de los usuarios, la posibilidad de consulta electrónica de su información clínica registrada en la plataforma de marras, obteniendo previamente los respectivos permisos de corroboración de su identidad digital.

La efectuada mención a las bases normativas elementales de la Historia Clínica Electrónica, apuntan a que la reglamentación de la forma de recabar el consentimiento informado en la telemedicina, debería necesariamente apoyarse en dichos cimientos normativos; mediante la implementación de un sistema que permita al usuario o paciente mantener acceso con facilidad el registro de todas las consultas empleadas en dicha modalidad telemática asistencia; y como derivado, de los alcances del consentimiento informado que haya brindado. En tal sentido, se deberá prever con claridad meridiana y debida especificidad el momento y forma en la que, durante la "teleconsulta" el paciente manifiesta que ha comprendido los alcances de la indicación efectuada por el médico, y si acepta o rechaza la misma; a fin de que dicho extremo sea consignado en la historia clínica en un modo que brinde certeza jurídica a ambas partes.

Eventualmente, y siempre bajo el respeto irrestricto de la noción de "datos sensibles" de la que establece la Ley $\mathrm{N}^{\mathrm{o}} 18.331$, con sus ineludibles caracteres de reserva y confidencialidad a rajatabla en el manejo de la información clínica; podría admitirse que la consulta realizada a través de telemedicina sea grabada como un archivo de audio, imagen y/o video e incorporada a la historia clínica electrónica del usuario. Dicho registro importará una doble autorización y/o consentimiento expreso por parte del paciente: el de aceptación de que se proceda a incorporar el registro en soporte intangible de la consulta, y el consentimiento informado a los efectos clínicos propiamente.

\section{Conclusiones.}

La telemedicina constituye un gran avance, en aspectos prácticos, para el desarrollo de prestaciones sanitarias, facilitando la cercanía del paciente con respecto a los profesionales médicos. Por otra parte, es una herramienta que se vincula con el principio bioético de justicia, en tanto provee posibilidades de acceso a la atención en salud de calidad, en condiciones de igualdad para los diferentes usuarios del sistema de salud, con independencia del lugar donde residan. 
En definitiva, con independencia de la situación actual de pandemia, se trata de un recursos indudablemente valioso que permanecerá tras la superación de los estragos generados por el COVID-19; y que seguramente aún no ha alcanzado el máximo de sus potencialidades.

No obstante, como todo progreso técnico y material, debe encaramarse en un marco regulatorio que permita proteger a los usuarios en el goce de sus derechos. En tal sentido la incipiente regulación de la Ley $\mathrm{N}^{\mathrm{o}} 19.869$ constituye un importante avance en dicha línea, el cual debe ser complementado con otros cuerpos legales preexistentes, a fin de brindar una protección más completa; conforme a los desarrollos que hemos efectuado

Los servicios asistenciales brindados a través de telemedicina debe instituirse bajo la égida de los principios especiales consagrados por el artículo 3 la Ley $\mathrm{N}^{\circ} 19.869$, añadiéndose además la importancia del deber de información, el consentimiento informado y la responsabilidad institucional en el manejo de la información sanitaria del usuario; ya que la infracción o proceder negligente con respecto a alguno de estos aspectos, es probable fuente generadora de responsabilidad civil para los prestadores.

Con relación al consentimiento informado, se aprecia que el reciente cuerpo normativo referido, en líneas generales se afianza en nociones preexistentes regentes en la materia, y que derivan del principio de autonomía, recogido en sendos instrumentos internacionales y nacionales. No obstante, se advierte una necesidad de adecuación en sede de menores e incapaces, teniendo en especial consideración la noción de autonomía progresiva, que en definitiva debe traducirse como una aplicación del principio de respeto por la dignidad humana.

Respecto de la modalidad en la forma de recabar u obtener el consentimiento informado "a distancia", ante la ausencia se reitera que las alternativas para su regulación puede legítimamente basarse y adaptarse las previsiones vigentes en materia de certificado electrónico, firma electrónica e historia clínica electrónica; ya que se trata de modalidades de registro de información con valor auténtico, de medio probatorio hábil y que brindan un importante grado de certeza jurídica, a pesar de su intangibilidad.

Nuestra última reflexión sobre el particular, se circunscribe al hecho de que el mundo recorre un camino, prácticamente irreversible, hacia el paulatino abandono de las formas documentales clásicas, respaldadas en soporte papel, por la sustitución de medios informáticos o virtuales. Para que este cambio sea conceptualizado como evolutivo y no involutivo, no depende únicamente la generación de una mayor comodidad para el usuario; sino que el ejercicio de dicha ventaja, en ningún caso, importe la violación de sus 
derechos fundamentales ni la pérdida de beneficios de relevancia, que le ofrece el sistema tradicional.

\section{Referencias normativas}

UNESCO. Declaración Universal sobre Bioética y Derechos Humanos de 19/10/2005.

URUGUAY. Ley $\mathrm{N}^{\circ} 17.823$ de 17/09/2004 (Código de la Niñez y de la Adolescencia). Publicación en Diario Oficial: 14/09/2008.

URUGUAY. Ley No 18.331 de 11/08/2008. Publicación en Diario Oficial: 18/08/2008.

URUGUAY. Ley No 18.335 de 15/08/2008. Publicación en Diario Oficial: 26/08/2008.

URUGUAY. Ley No 18.473 de 03/04/2009. Publicación en Diario Oficial: 21/04/2009.

URUGUAY. Ley No 18.600 de 21/09/2009. Publicación en Diario Oficial: 05/11/2009.

URUGUAY. Ley No 18.719 de 27/12/2010. Publicación en Diario Oficial: 05/01/2011.

URUGUAY. Ley No 19.286 de 25/09/2014. Publicación en Diario Oficial: 17/10/2014.

URUGUAY. Ley No 19.355 de 19/12/2015. Publicación en Diario Oficial: 30/12/2015.

URUGUAY. Ley No 19.529 de 24/08/2017. Publicación en Diario Oficial: 19/09/2017.

URUGUAY. Ley N 19.670 de 15/10/2018. Publicación en Diario Oficial: 25/10/2018.

URUGUAY. Ley No 19.869 de 02/04/2020. Publicación en Diario Oficial: 15/04/2020.

URUGUAY. Ley No 19.924 de 18/12/2020. Publicación en Diario Oficial: 30/12/2020.

URUGUAY. Decreto No 274/010 de 08/09/2010. Publicación en Diario Oficial: 16/09/2010.

URUGUAY. Decreto No 242/017 de 31/08/2017. Publicación en Diario Oficial: 07/09/2017

URUGUAY. Decreto No 122/019 de 29/04/2019. Publicación en Diario Oficial: 13/05/2019.

URUGUAY. Decreto No 158/019 de 03/06/2019. Publicación en Diario Oficial: 12/06/2019.

URUGUAY. Decreto No 93/020 de 13/03/2020. Publicación en Diario Oficial: 23/03/2020. 


\section{BIBLIOGRAFÍA CONSULTADA}

BERRO, G. y ADRIASOLA, G. (2013) Confidencialidad. En BERRO, G. Medicina Legal. Derecho Médico y aspectos bioéticos. FCU.

ESCOBAR SUHR, V. y ANDRADE SOSA, D. (2020) La telemedicina en el marco de las relaciones de consumo. En AA.VV. El Derecho Comercial frente a las crisis globales. Sociedades - Contratos Concursos. FCU. -

GÓMEZ SÁNCHEZ, Y. (2016) Derecho a no saber. En AA.VV Enciclopedia de Bioderecho y Bioética https://enciclopedia-bioderecho.com/voces/102

GIL YACOBAZZO, J. y VIEGA RODRÍGUEZ, M. (2018) Historia clínica electrónica: confidencialidad y

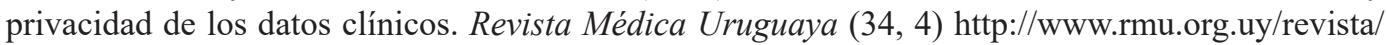
proximo/rmu34-4_921-gil-historia.pdf

ORDOQUI CASTILLA, G. (2012). Consentimiento Informado. Ley 18335 y Decreto 274/10. Ediciones del Foro.

Fecha de recepción: 26 de abril 2021 Fecha de aceptación: 2 de mayo 2021 